\title{
A Descriptive Study of Emergency Department Visits Within 30 Days of Discharge
}

\author{
Hyeanji Kim ${ }^{1}$, Seung Jun Han ${ }^{2,3,}$, Jae Hyun Lee ${ }^{2,3}$, Jin Lim ${ }^{2,3}$, Sung do Moon ${ }^{2,3}$, Hongran Moon ${ }^{2,3}$, Seo-Young Lee ${ }^{2,3}$, \\ Sock-Won Yoon ${ }^{2,3}$, Hee-Won Jung ${ }^{4, *}$ \\ ${ }^{1}$ Regional Emergency Medical Center, Seoul National University Hospital, Seoul, Korea \\ ${ }^{2}$ Department of Internal Medicine, Seoul National University Hospital, Seoul, Korea \\ ${ }^{3}$ Hospital Medicine Center, Seoul National University Hospital, Seoul, Korea \\ ${ }^{4}$ Division of Geriatrics, Department of Internal Medicine, Asan Medical Center, University of Ulsan College of Medicine, Seoul, Korea
}

Corresponding Author:

Seung Jun Han, MD

Department of Internal Medicine,

Seoul National University Hospital, 101

Daehak-ro, Jongno-gu, Seoul 03080,

Korea

E-mail: hsjjam@naver.com

ORCID:

https://orcid.org/0000-0001-8960-3542

Hee-Won Jung, $\mathrm{MD}$, $\mathrm{PhD}$

Division of Geriatrics, Department of

Internal Medicine, Asan Medical

Center, University of Ulsan College of

Medicine, 88, Olympic-ro 43-gil,

Songpa-gu, Seoul05556, Korea

E-mail: dr.ecsta@gmail.com

ORCID:

https://orcid.org/0000-0002-2583-3354
Background: Unnecessary emergency department (ED) visits are a crucial consideration in discharge planning for acutely admitted patients. This study aimed to identify the reasons for unnecessary visits to the ED within 30 days of discharge from a medical hospitalist unit. Methods: We performed a retrospective review of patients discharged in 2018 from a medical unit of tertiary teaching hospital in Korea. The authors discussed in-depth and determined whether or not an ED visit was unnecessary, and further classified the causes of unnecessary visits into three categories. Results: The mean age of the patients was 62.9 years (range, 15-99 years), and among the 1,343 patients discharged from the unit, 720 (53.6\%) were men. Overall, 215 patients (16.0\%) visited the ED within 30 days after discharge; among them, $16.3 \%$ were readmitted. Of the 215 cases of ED visits within 30 days after discharge, 57 (26.5\%) were considered unnecessary. Of these, 30 (52.6\%) were categorized as having failed care transition, $15(26.3 \%)$ had unestablished care plans for predictable issues, and $12(21.1 \%)$ had insufficient patient education. Conclusion: A substantial number of short-term ED visits by discharged multimorbid or older medical patients were considered unnecessary. Discharging patients with a thorough discharge plan is essential to avoid unnecessary ED visits.

Key Words: Patient discharge, Patient readmission, Geriatrics, Multimorbidity, Hospital medicine

Received: July 10, 2021

Revised: October 18, 2021

Accepted: October 19, 2021

*Both authors contributed equally to

this work.

\section{INTRODUCTION}

In Korea, where geographic and financial accessibilities to tertiary hospitals are high, overcrowding of the emergency department (ED) of tertiary medical institutions is a serious problem. Such overcrowding, especially in the ED, leads to poor patient outcomes, increased length of stay, and increased risk of infectious dis- ease transmission. ${ }^{1-4)}$ The rates of unplanned readmission of hospitalized patients after discharge range between $10 \%$ and $25 \%{ }^{5-10)}$ with most patients readmitted through the ED. Thus, discharge planning to minimize unnecessary post-discharge ED visits should be considered an important medical issue to reduce $\mathrm{ED}$ overcrowding and consequent readmissions.

With the increasing prevalence of chronic diseases owing to ag- 
ing, many patients have multimorbidities. ${ }^{11-13)}$ The risks of readmission and ED visits are high among older or multimorbid patients. Hence, eliminating unnecessary hospital visits among these patients is a priority. ${ }^{14-18)}$ Since the launch of the hospitalist system, older or multimorbid patients are often treated in hospital units by hospitalists who provide generalized medical care. ${ }^{5,6)}$ Studies of medical hospitalist units in tertiary teaching hospitals in Korea have reported that the mean age of hospital inpatients is $>60$ years, that most patients have multimorbidities, and that the average Charlson Comorbidity Index score is 6 or more. ${ }^{5)}$ Hence, the readmission of patients from hospitalist units is of crucial concern when considering post-discharge $\mathrm{ED}$ visits.

To our knowledge, no published data have yet described the details and causes of ED visits after acute admission to hospitalist units in Korea. This study aimed to identify reasons for visiting the ED within 30 days of discharge and describe the reasons for unnecessary ED visits in multimorbid or older medical patients. We assessed cases in detail and proposed how physicians might prevent unnecessary ED visits, which could not only reduce ED overcrowding but also minimize unnecessary use of medical resources. $^{19)}$

\section{MATERIALS AND METHODS}

\section{Study Design and Participants}

This retrospective database study collected information on ED visits of patients who were discharged from the hospitalist unit of a tertiary teaching hospital in Korea in 2018. The medical hospitalist unit services older or multimorbid inpatients as described in previous studies from the same unit. ${ }^{5,6)}$ We then reviewed in detail medical records of patients who visited the ED within 30 days of discharge.

The study protocol was reviewed and approved by the Institutional Review Board of the Seoul National University Hospital (No. 2021-070-1180), which approved a waiver of consent because of the retrospective nature of the study. The study protocol conformed to the ethical guidelines of the 1975 Declaration of Helsinki.

\section{Determination and Classification of Unnecessary ED Visits}

Previous studies have proposed criteria for determining unnecessary ED visits based on diagnostic or procedural codes. ${ }^{20,21)}$ However, criteria based on codes are insufficient to identify the cause of each case or suggest countermeasures. Thus, in this study, a roundtable discussion was performed with all authors who were board-certified internists and currently working in the ED or medical hospitalist units in the same hospital to discuss each case in depth. First, unnecessary ED visits were determined based on the following criteria.

(1) Primary care treatable: A patient visited the ED with symptoms that were not obvious or mild enough to be managed by the primary outpatient clinic of the community.

(2) Preventable: If a patient's symptoms were reported during the index hospitalization, an appropriate plan was omitted at the time of discharge.

(3) Hospice care suitable: If a patient's symptoms were worse than before or new, but could have been predicted while the underlying disease was progressing, and for which hospice care was recommended for patients during the index hospitalization.

Second, unnecessary ED visits were classified according to causes:

(1) Failure of care transitions: Cases that returned to the ED owing to unmet medical or care needs after discharge to the community or that were admitted to medical institutions in the community. This category also included issues regarding discordance between physicians and patients regarding the therapeutic goal of index hospitalizations leading to ED visits.

(2) Unestablished care plans for predictable issues: This category included unnecessary ED visits associated with previous discharge planning, such as predictable medical or caregiving issues after index discharge regarding symptoms (e.g., pain, dyspnea) of the underlying medical conditions.

(3) Insufficient patient education: This category included issues such as education for the management of feeding tubes, drainage tubes, or intravenous catheters, as well as the administration of certain medications.

\section{Statistical Analysis}

Data are expressed as mean \pm standard deviation or number (percentage), unless stated otherwise. Independent t-test and Pearson $\chi^{2}$ test were used to compare continuous and categorical variables, respectively, between patients who visited the ED and those who did not. We considered two-sided p-values $<0.05$ to be statistically significant. IBM SPSS Statistics for Windows version 23.0 (IBM Corp., Armonk, NY, USA) was used for all statistical analyses.

\section{RESULTS}

\section{Clinical Characteristics of Index Hospitalizations}

A total of 1,343 patients were discharged from the medical hospitalist unit during the study period. Their mean age was 62.9 years (range, 15-99 years); 720 patients (53.6\%) were men, and 323 pa- 
tients $(24.1 \%)$ were admitted to the ED. A total of 1,052 patients (78.3\%) were initially admitted owing to cancer-related problems. Excluding patients with cancer, the common reasons for admission were respiratory tract infection, liver disease, and kidney disease. The mean length of stay was 11.3 nights, and 57 patients (4.2\%) were transferred to long-term care hospitals after discharge.

A total of 215 patients (16.0\%) visited the ED within 30 days of discharge. Among them, 16.3\% were admitted from the ED to the ward. The mean age of patients did not differ significantly between those who did and did not visit the ED. Compared to those in the non-visiting group, patients in the visiting ED group had higher proportions of men and patients admitted to the ED for index hospitalization (63.3\% vs. $51.8 \%$ and $34.0 \%$ vs. $22.3 \%$; $p=0.002$ and $\mathrm{p}<0.001$, respectively). Patients in the visiting ED group also had longer hospital stays during the index hospitalization than those in the non-visiting group (mean, 13.4 nights vs. 10.9 nights; $\mathrm{p}=0.001$ ). The proportions of patients with cancer or those who were transferred to long-term care hospitals did not differ between the two groups (Table 1).

\section{Clinical Characteristics and Causes of Unnecessary ED Visits}

Of the 215 visits to the ED within 30 days of discharge, 57 (26.5\%) were determined to be unnecessary (40 primary care treatable, 12 preventable, and 5 hospice care suitable). There were fewer men in the unnecessary visiting group than in the necessary visiting group ( $49.1 \%$ vs. $68.4 \%$; $=0.010$ ), and no significant differences in age, route of index hospitalization, proportion of patients with cancer, length of hospital stay, and proportion of patients transferred were noted between the two groups (Table 2).

Categorization of unnecessary ED visits according to causes revealed 30 (52.6\%) visits for failure of care transition, 15 (26.3\%) for unestablished care plans for predictable issues, and $12(21.1 \%)$ for insufficient patient education (Fig. 1).

\section{First cause: failure of care transitions}

This category was the most common reason for unnecessary ED visits. Most cases in this category ( 24 of 30 visits) were treatable by primary care institutions. The cases in this category largely involved communication issues related to discharge planning or insufficient information about the local health system's coping capabilities. Several patients wanted parenteral nutrition, paracentesis, medications to control non-serious symptoms, and reassurance regarding insignificant symptoms. For example, a 49-year-old male patient returned to the ED 3 weeks after transfer seeking a prescription for laxatives. This category also included patients and their caregivers who were dissatisfied with the local medical insti-

Table 1. Clinical characteristics of the study population

\begin{tabular}{lccr}
\hline Variable & Non-visiting & Visiting ED & p-value \\
\hline Number of patients & $1,128(84.0)$ & $215(16.0)$ & 0.978 \\
Age (y) & $62.9 \pm 13.6$ & $62.8 \pm 13.2$ & $136(63.3)$ \\
Sex, male & $584(51.8)$ & $73(34.0)$ & 0.002 \\
Index hospitalization through the ED & $250(22.3)$ & $176(81.9)$ & 0.001 \\
Malignancy-associated problems & $876(77.7)$ & $13.4 \pm 9.3$ & 0.171 \\
Length of stay (nights) & $10.9 \pm 10.1$ & $6(2.8)$ & 0.001 \\
Transfer to LTCHs & $51(4.5)$ & 0.248 \\
\hline
\end{tabular}

Values are presented as number (\%) or mean \pm standard deviation.

ED, emergency department; LTCH, long-term care hospital.

Table 2. Clinical characteristics of patients who visited the ED within 30 days of discharge

\begin{tabular}{lccc}
\hline Variable & Necessary & Unnecessary & p-value \\
\hline Number of patients & $158(73.5)$ & $57(26.5)$ & 0.136 \\
Age (y) & $63.6 \pm 12.7$ & $60.6 \pm 14.7$ & 0.010 \\
Sex, male & $108(68.4)$ & $28(49.1)$ & 0.274 \\
Index hospitalization through ED & $57(36.1)$ & $16(28.1)$ & 0.142 \\
Malignancy-associated problems & $133(84.2)$ & $43(75.4)$ & 0.680 \\
Length of stay (nights) & $13.5 \pm 9.3$ & $12.9 \pm 9.3$ & $0.657^{*}$ \\
Transfer to LTCHs & $4(2.5)$ & $2(3.5)$ & \\
\hline
\end{tabular}

Values are presented as number (\%) or mean \pm standard deviation. ED, emergency department; LTCH, long-term care hospital.

*Fisher exact test. 

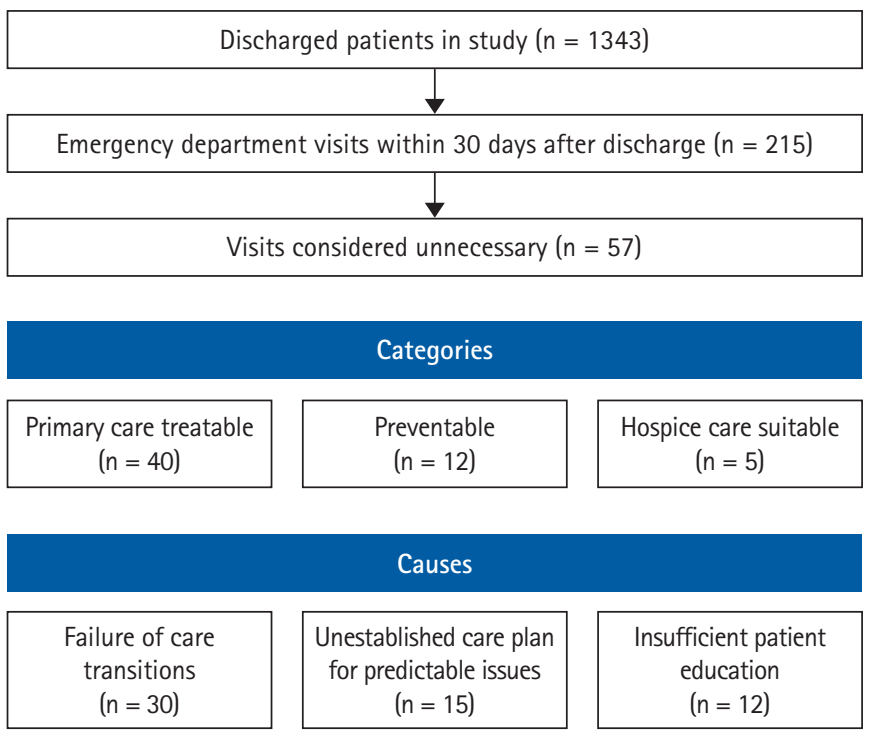

Fig. 1. Classification according to causes of unnecessary emergency department visits.

tutions who instead visited the ED of a tertiary hospital seeking admission. In this category, three patients returned to the ED after being discharged home and were eventually transferred to a medical institution at which hospice care was available.

\section{Second cause: unestablished care plans for predictable issues}

The most common reason for ED visits in this category was deficient analgesics for predictable pain ( 9 of 15 visits), such as breakthrough cancer pain. A patient revisited the ED complaining of abdominal pain only 3 days after discharge. The patient had stage IV intrahepatic cholangiocarcinoma and was discharged after undergoing biopsy of the newly detected neoplasm found on computed tomography. Since the patient complained of persistent pain in the right upper quadrant during hospitalization, it was predictable that the pain would continue even after discharge. There were also several cases of ED revisits owing to diet-related problems (3 of 15 visits). For instance, a 58-year-old man was brought to the ED because of seizures. During index hospitalization, the patient had a seizure for the first time and was prescribed an antiepileptic drug. However, pills were prescribed at the time of discharge despite the patient having difficulty swallowing. As the patient could not take oral medication, the blood level of the antiepileptic drug decreased, resulting in more seizures.

\section{Third cause: insufficient patient education}

A total of 12 patients returned to the ED owing to a lack of training in drug administration and management of feeding or drainage tubes. One patient also visited the ED because of hypoglycemia. During the previous hospitalization, the patient started taking in- sulin for the first time and thus was not familiar with insulin administration. Another patient returned to the ED because the percutaneous transhepatic biliary drainage (PTBD) catheter had disconnected from the drainage bag. The patient was discharged in the morning and had to return to the ED within several hours. The patient returned home immediately after the healthcare staff reconnected the PTBD catheter to the drainage bag. Most cases in this category ( 10 of 12 ) were treatable by primary care.

\section{DISCUSSION}

Consistent with the literature, the results of this study revealed that a substantial number of patients returned to the ED shortly after discharge. Of these, $26.5 \%$ of ED visits were unnecessary. Unnecessary ED visits cost patients, consume their physical strength, and risk the acquisition of infectious diseases. More importantly, unnecessary ED visits may lead to wasted medical resources and prevent the efficient use of these resources for patients who require emergency treatment. In this study, we classified the causes of unnecessary ED visits after discharge into three categories.

In this study population, the most common type of unnecessary ED visits was primary care treatable, often caused by a failure of transition care. It is essential to link patients to community medical institutions at or before discharge so that they can visit primary or secondary medical institutions rather than tertiary hospitals for mild symptoms. It is also important to create a referral detailing the patient's condition for local primary or secondary medical institutions. Patients also return to the ED after discharge because of a lack of communication between the staff of medical institutions. If a patient is discharged from a tertiary hospital and transferred to a nursing home or another hospital, the attending physician must fully explain the patient's condition to the medical staff at the hospital. For instance, if no additional treatment plan and hospice care are needed, ED visits for worsening medical conditions can be a futile process. Patients may experience unnecessary suffering from being repeatedly transferred to the ED, even when death is imminent. Sharing information and ensuring two-way open communication between medical service providers are important for reducing unnecessary ED visits. ${ }^{22,23)}$ In addition, a system that allows hospitals to share medical records for a certain period before and after transfer is also worth considering.

Patient factors often negate efforts for effective care transitions. This may be because of current medical delivery systems in Korea. Patients in Korea can receive treatment at a tertiary hospital for a similar fee, even for common mild chronic disease, with a simple request from the primary clinic. ${ }^{24)}$ Medical institutions at each level are trying to attract patients through competition rather than co- 
operation. Most patients, regardless of their degree of discomfort, tend to seek treatment at large tertiary hospitals. A similar trend has been reported in emergency care. ${ }^{25,26)}$ Therefore, successful care transitions should always also inform patients about the capabilities of local primary or secondary medical institutions. Attending physicians must also explain to patients the disease course and prognosis and provide a list of indications for when he or she should visit the ED of the tertiary hospital at which they had previously received treatment. The patients in this study tended to visit the ED even for mild symptoms that did not require treatment at a tertiary hospital. When a patient is discharged from a tertiary medical institution, the attending physician may instruct the patient to visit a local primary or secondary medical institution for minor problems. ${ }^{27,28)}$

Predicting and responding to potential health-related situations in patients are important roles of attending physicians upon discharge. Unless told how to properly cope with their situation, patients will likely return to the ED every time unexpected symptoms occur. When a meticulous care plan is not available, patients will likely return to the $\mathrm{ED}$ after discharge for inappropriate issues such as hydration, additional nutritional supplementation, or drug prescription. When a physician prescribes discharge medications, it is necessary to follow a process that reflects the opinions of pharmacists, nurses, caregivers, and patients. ${ }^{29-32)}$

Patient education must be provided to empower their self-care ability upon discharge. ${ }^{27,29,32)}$ Such education may include information on daily life, such as eating and bathing, or medical information such as the name, dose, and method of taking medications. In particular, healthcare professionals must educate patients who are discharged from the hospital with indwelling medical devices such as urinary catheters, nasogastric tubes, or PTBD on proper management procedures. Training these patients to adjust their insulin dose or manage drainage tubes would avoid visits to the ED. If education encompassing instructions for patients who are discharged from acute hospitals could be reimbursed, unnecessary ED visits associated with this issue might be alleviated.

Discharging patients from hospitals is a complex process, as observed for each case in this study. Meticulous and individualized discharge plans may reduce unnecessary post-discharge ED visits. Previous studies have proposed various approaches to improve the discharge process and reduce unnecessary ED visits, including pre-discharge interventions (including discharge planning, patient education, and scheduling follow-up appointments) or post-discharge interventions (follow-up phone calls after discharge, communication by telemedicine, etc.). ${ }^{33-35)}$ Bridging interventions, such as transition coaches and continuity of care between inpatient and outpatient departments, may also be helpful. Reducing unnec- essary ED visits may improve both patient quality of life and the financial status of the healthcare system. Furthermore, the burden on medical personnel may decrease.

The findings of this study should be considered in light of the following limitations. First, the study sample was limited to data from the medical hospitalists-operated unit of a tertiary hospital, in which most patients were older or had serious underlying diseases such as cancer. The results of this study might be difficult to apply to primary or secondary local hospital situations. Second, cases of ED visits other than the hospital where the patients were discharged were not investigated. Third, this study depended on the accuracy of the documented data, which was retrospective and administrative in nature. Finally, although there have been suggestions on ways to reduce ED visits, none of them were assessed for their efficacy in this descriptive study.

In conclusion, a significant number of short-term ED visits after the discharge of multimorbid or older medical patients were unnecessary. Comprehensive discharge procedures, including care transition, planning for predictable issues, and patient education, are essential. Further interventional and prospective studies are needed to investigate the effects of discharge interventions on $\mathrm{ED}$ visits.

\section{ACKNOWLEDGMENTS}

\section{CONFLICT OF INTEREST}

The researchers claim no conflicts of interest.

\section{FUNDING}

None.

\section{AUTHOR CONTRIBUTION}

Conceptualization, HK; Data curation, HK, SJH, JHL, SM, HM, SYL, SWY, HWJ; Formal analysis, HK, SJH, JHL, SM, HM, SYL, SWY, HWJ; Investigation, SJH; Methodology, SJH, HWJ; Software, SJH; Visualization, HK; Writing - original draft, HK, SJH; Writing - review \& editing, SJH.

\section{REFERENCES}

1. Di Somma S, Paladino L, Vaughan L, Lalle I, Magrini L, Magnanti M. Overcrowding in emergency department: an international issue. Intern Emerg Med 2015;10:171-5.

2. Trzeciak S, Rivers EP. Emergency department overcrowding in the United States: an emerging threat to patient safety and public health. Emerg Med J 2003;20:402-5.

3. Kim YJ, Choe JY, Kwon KT, Hwang S, Choi GS, Sohn JH, et al. 
How to keep patients and staff safe from accidental SARS-CoV-2 exposure in the emergency room: lessons from South Korea's explosive COVID-19 outbreak. Infect Control Hosp Epidemiol 2021;42:18-24.

4. Cho SY, Kang JM, Ha YE, Park GE, Lee JY, Ko JH, et al. MERS$\mathrm{CoV}$ outbreak following a single patient exposure in an emergency room in South Korea: an epidemiological outbreak study. Lancet 2016;388:994-1001.

5. Han SJ, Jung HW, Oh DY, Lee JH, Moon SD, Lee S, et al. Comparisons of clinical outcomes between weekday-only and fulltime, 24-hour/7-day coverage hospitalist systems. J Korean Med Sci 2020;35:e117.

6. Lee JH, Jung HW, Jang IY, do Moon S, Lee S, Han SJ. Anticholinergic cognitive burden as a predictive factor for in-hospital mortality in older patients in Korea. Ann Geriatr Med Res 2020;24:20-6.

7. Jang JG, Ahn JH. Reasons and risk factors for readmission following hospitalization for community-acquired pneumonia in South Korea. Tuberc Respir Dis (Seoul) 2020;83:147-56.

8. Kim YM, Lee T, Lee HJ, Yang YL, Oh EG. Readmission of highrisk discharged patients at a tertiary hospital in Korea. J Healthc Qual 2019;41:e30.-7.

9. Donze J, Lipsitz S, Bates DW, Schnipper JL. Causes and patterns of readmissions in patients with common comorbidities: retrospective cohort study. BMJ 2013;347:f7171.

10. Elixhauser A, Steiner C. Readmissions to U.S. hospitals by diagnosis, 2010. In: Healthcare Cost and Utilization Project (HCUP) statistical briefs. Rockville, MD: Agency for Healthcare Research and Quality; 2006.

11. Jang IY, Lee HY; Lee E; 50th Anniversary Committee of Korean Geriatrics Society. Geriatrics fact sheet in Korea 2018 from national statistics. Ann Geriatr Med Res 2019;23:50-3.

12. Jung HW, Kim KI. Multimorbidity in older adults. J Korean Geriatr Soc 2014;18:65-71.

13. Marengoni A, Angleman S, Melis R, Mangialasche F, Karp A, Garmen A, et al. Aging with multimorbidity: a systematic review of the literature. Ageing Res Rev 2011;10:430-9.

14. Aminzadeh F, Dalziel WB. Older adults in the emergency department: a systematic review of patterns of use, adverse outcomes, and effectiveness of interventions. Ann Emerg Med 2002;39: 238-47.

15. Wofford JL, Schwartz E, Timerding BL, Folmar S, Ellis SD, Messick CH. Emergency department utilization by the elderly: analysis of the National Hospital Ambulatory Medical Care Survey. Acad Emerg Med 1996;3:694-9.

16. Zekry D, Loures Valle BH, Graf C, Michel JP, Gold G, Krause $\mathrm{KH}$, et al. Prospective comparison of 6 comorbidity indices as predictors of 1-year post-hospital discharge institutionalization, readmission, and mortality in elderly individuals. J Am Med Dir Assoc 2012; 13:272-8.

17. van Walraven C, Dhalla IA, Bell C, Etchells E, Stiell IG, Zarnke K, et al. Derivation and validation of an index to predict early death or unplanned readmission after discharge from hospital to the community. CMAJ 2010;182:551-7.

18. Librero J, Peiro S, Ordinana R. Chronic comorbidity and outcomes of hospital care: length of stay, mortality, and readmission at 30 and 365 days. J Clin Epidemiol 1999;52:171-9.

19. Duseja R, Bardach NS, Lin GA, Yazdany J, Dean ML, Clay TH, et al. Revisit rates and associated costs after an emergency department encounter: a multistate analysis. Ann Intern Med 2015;162:750-6.

20. Billings J, Parikh N, Mijanovich T. Emergency department use in New York City: a substitute for primary care? Issue Brief (Commonw Fund) 2000;(433):1-5.

21. Jeffery MM, Bellolio MF, Wolfson J, Abraham JM, Dowd BE, Kane RL. Validation of an algorithm to determine the primary care treatability of emergency department visits. BMJ Open 2016;6:e011739.

22. Hesselink G, Schoonhoven L, Barach P, Spijker A, Gademan P, Kalkman C, et al. Improving patient handovers from hospital to primary care: a systematic review. Ann Intern Med 2012;157: 417-28.

23. Graumlich JF, Novotny NL, Nace GS, Aldag JC. Patient and physician perceptions after software-assisted hospital discharge: cluster randomized trial. J Hosp Med 2009;4:356-63.

24. Kwon S. Payment system reform for health care providers in Korea. Health Policy Plan 2003; 18:84-92.

25. Lee JY, Jo MW, Yoo WS, Kim HJ, Eun SJ. Evidence of a broken healthcare delivery system in Korea: unnecessary hospital outpatient utilization among patients with a single chronic disease without complications. J Korean Med Sci 2014;29:1590-6.

26. Kim AM, Cho S, Kim HJ, Jung H, Jo MW, Lee JY, et al. Primary care patients' preference for hospitals over clinics in Korea. Int J Environ Res Public Health 2018;15:1119.

27. DeVries A, Li CH, Oza M. Strategies to reduce nonurgent emergency department use: experience of a Northern Virginia Employer Group. Med Care 2013;51:224-30.

28. Flores-Mateo G, Violan-Fors C, Carrillo-Santisteve P, Peiro S, Argimon JM. Effectiveness of organizational interventions to reduce emergency department utilization: a systematic review. PLoS One 2012; 7:e35903.

29. Koehler BE, Richter KM, Youngblood L, Cohen BA, Prengler ID, Cheng D, et al. Reduction of 30-day postdischarge hospital readmission or emergency department (ED) visit rates in high- 
risk elderly medical patients through delivery of a targeted care bundle. J Hosp Med 2009; 4:211-8.

30. Reidt SL, Larson TA, Hadsall RS, Uden DL, Blade MA, Branstad R. Integrating a pharmacist into a home healthcare agency care model: impact on hospitalizations and emergency visits. Home Healthc Nurse 2014;32:146-52.

31. Rich MW, Beckham V, Wittenberg C, Leven CL, Freedland KE, Carney RM. A multidisciplinary intervention to prevent the readmission of elderly patients with congestive heart failure. $\mathrm{N}$ Engl J Med 1995;333:1190-5.

32. Jack BW, Chetty VK, Anthony D, Greenwald JL, Sanchez GM, Johnson AE, et al. A reengineered hospital discharge program to decrease rehospitalization: a randomized trial. Ann Intern Med 2009; 150:178-87.
33. Althaus F, Paroz S, Hugli O, Ghali WA, Daeppen JB, Peytremann-Bridevaux I, et al. Effectiveness of interventions targeting frequent users of emergency departments: a systematic review. Ann Emerg Med 2011;58:41-52.

34. Kumar GS, Klein R. Effectiveness of case management strategies in reducing emergency department visits in frequent user patient populations: a systematic review.J Emerg Med 2013;44:717-29.

35. Racine AD, Alderman EM, Avner JR. Effect of telephone calls from primary care practices on follow-up visits after pediatric emergency department visits: evidence from the Pediatric Emergency Department Links to Primary Care (PEDLPC) randomized controlled trial. Arch Pediatr Adolesc Med 2009;163:50511. 